Springer Japan KK 


\author{
M. Inaba, Y. Inaba
}

\title{
Androgenetic Alopecia
}

Modern Concepts of

Pathogenesis and Treatment

With 425 Illustrations, with 92 in Color 
Masumi Inaba, M.D.

Inaba Clinic

Department of Aesthetic Plastic Surgery

3-31-13 Asagaya-minami,

Suginami-ku, Tokyo, 166 Japan

Yoshikata Inaba, M.D.

Department of Dermatology

The Jikei University School of Medicine

3-25-8 Nishi-shinbashi,

Minato-ku, Tokyo, 105 Japan

and

Department of Dermatology

Inaba Aesthetic Plastic Surgery

3-31-13 Asagaya-minami,

Suginami-ku, Tokyo, 166 Japan

ISBN 978-4-431-67040-7 ISBN 978-4-431-67038-4 (eBook)

DOI 10.1007/978-4-431-67038-4

Printed on acid-free paper

(C) Springer Japan 1996

Originally published by Springer-Verlag Tokyo Berlin Heidelberg New York in 1996

This work is subject to copyright. All rights are reserved, whether the whole or part of the material is concerned, specifically the rights of translation, reprinting, reuse of illustrations, recitation, broadcasting, reproduction on microfilms or in other ways, and storage in data banks.

The use of registered names, trademarks, etc. in this publication does not imply, even in the absence of a specific statement, that such names are exempt from the relevant protective laws and regulations and therefore free for general use.

Product liability: The publisher can give no guarantee for information about drug dosage and application thereof contained in this book. In every individual case the respective user must check its accuracy by consulting other pharmaceutical literature.

Typesetting: Best-set Typesetter Ltd., Hong Kong 


\section{Foreword}

Until now, the general belief has been that premature baldness (male pattern baldness: M.P.B., or androgenetic alopecia) is a hereditary condition that progresses with age and for which there is no cure. If the father is bald, it is assumed that the child may also be destined to be bald sometime in the future.

The existing theory throughout the world is that the central portion of the hair follicle is the hair root, and hair can never regrow if the hair root has been removed. Dr. Inaba has introduced a new theory that the central portion of the hair follicle is not only the hair root but the upper isthmal portion close to the duct opening of the sebaceous gland. This is a totally new understanding of the hair growth mechanism and the hair cycle. In his experience in the surgical removal of the sweat glands, which also removes the hair root, Dr. Inaba has found increasing evidence that hair regrows if the sebaceous gland is left intact.

The prevailing belief is that the male hormone acts directly on the hair root to cause male pattern baldness. However, Dr. Inaba has found that the male hormone is affected by an enzyme in the sebaceous gland which exercises a secondary effect on the hair root. An important factor in baldness is the size of the sebaceous gland; the relative size of the gland is influenced by diet and animal fats. Thus, baldness is not primarily a hereditary condition but rather a result of the combination of the hereditary strength of the hormone and individual dietary habits.

This new theory helped Dr. Inaba to develop a treatment for baldness based on the prevention of the enlargement of the sebaceous gland. The treatment includes appropriate diet, the use of a shampoo containing an enzyme-suppression agent, and trichogenous agents. We sincerely hope that this book will help many people to overcome or retard their premature baldness.

Robert M. Nakamura, M.D.

Chairman Emeritus

Department of Pathology

Scripps Clinic and Research Foundation

La Jolla, CA 92037, USA 


\section{Foreword}

Studies in the physiology and pathology of hair, especially clinical research concerned with the growth of hair, have been minimal compared with studies carried out in other fields of medical science. Only a small number of scientists at a few research centers in the United States and Europe have done studies on the growth of hair.

Recently, however, alopecia has emerged as a concern of young people, mainly in Europe, the United States, and Japan, where a high standard of living is enjoyed. Consequently, there has been a sudden rush of studies of hair in the fields of dermatology, plastic surgery, and cosmetic surgery.

As a practitioner and as director of a small hair research center in Tokyo, Dr. Masumi Inaba, the principal author of this book, has carried out numerous studies of the physiology of hair. The regular observation of hair growth by means of a unique operative technique for relief from bromidrosis, which Dr. Inaba developed, well deserves acclaim as a landmark in the field.

Dr. Inaba has now published the result of his work on androgenetic alopecia, an endeavor that he has taken on as a lifetime study. I pay my deep respect to his arduous efforts resulting from reading a wide range of literature, profound observational practice, and knowledge derived from practical clinical work. I am reminded of the famous phrase, "Gradual approach to truth." I am convinced that Dr. Inaba's work published here will serve as an excellent basic reference text for medical practitioners and students in this field in the years to come.

Tai Ho Chung, M.D., Ph.D.

Director and Professor

Biomedical Research Laboratory

School of Medicine

Kyungpook National University

Taegu, Korea 


\section{Preface}

The recent progress of medicine is, in a word, remarkable. However, the stubborn problems of cancer and pathological human hair loss still elude the search for a final cure. The research that establishes such cures, it could be said, would merit a Nobel Prize.

The complexities of cancer continue to baffle dedicated research teams, but we all look forward fervently to an eventual end to this fearful disease. On the other hand, little attention is paid to the phenomenon of human baldness. Its onset in middle age, or sometimes much earlier, seems to provoke a certain sense of idle resignation, much as if to say that it is just one of the unavoidable hazards of growing older. And, after all, it does no actual harm in other than a cosmetic sense. In some societies a bald head is even considered a symbol of wisdom or virility.

However we elect to perceive it, the phenomenon of androgenetic alopecia, in particular, has been treated rather lightly by much of the medical profession. Any explanation of its causation, spun out by any researcher regarded as an expert, has been thoughtlessly accepted even if it is later found to be quite false. Erroneous notions about the causes of baldness have perhaps been among the leading reasons why basic research and treatment procedures have been delayed for so long. It is only quite recently that serious attention has been brought to bear on the problem.

The authors have conducted basic research studies on the phenomenon of baldness and have developed new treatment techniques over the past 20 years. One conclusion is that the common explanation of the human hair cycle is, in some respects, inaccurate and misleading. This conclusion has been made on the basis of some 10,000 clinical cases and a growing body of histological studies. Much of the histological research work was done with original thick-tissue specimens which provided threedimensional views of hair generation and regeneration.

As a result, the authors have formulated a new hypothesis to elucidate the phenomenon of human hair loss and regeneration. Research papers that led to this hypothesis have been published in a number of leading medical journals, both in Japan and in other countries. The key feature of the hypothesis is the major role played by the sebaceous gland in the complex process of hair loss and regeneration. Most recently, this hypothesis was presented in the authors' Human Body Odor published by Springer-Verlag.

The lifelong process of human hair generation and recession includes the phenomenon of baldness. Recent studies have indicated new treatment approaches to retard or prevent loss of hair which are quite different from those methods attempted in the past. Modern-day medical advances make it seem preposterous that men of less than 100 years of age should continue to lose their hair. It strikes the authors as strange indeed that the onset of baldness at 40 to 50 years of age is accepted by most men as regrettably inevitable. Some men, in fact, begin to grow bald as soon as their early 20 s. This seems even more unnatural. 
In light of anticipated future advances in medicine, this present book may be no more than a small first step toward the goal of a cure for baldness. It is, however, a step that is fully based on scientific studies, and we wish to commend the contents not only to men (and to some women) who fall victim to baldness but to anyone who has a serious interest in research within the field of dermatology.

Chapters 1-16 have been written with the basic research related to androgenetic alopecia very much in mind. Chapters 17-32 are intended to present in-depth research in the subject matter.

It is the authors' hope that the material presented herein will indeed inspire a renewed determination to conduct further research and development studies that will fully clarify the causative mechanism of male pattern baldness and other types of pathological hair loss and lead to permanent, fully effective remedies for prevention and cure.

Masumi Inaba, M.D.

Yoshikata Inaba, M.D. 


\section{Contents}

Foreword $\ldots \ldots \ldots \ldots \ldots \ldots \ldots \ldots \ldots \ldots \ldots \ldots \ldots \ldots \ldots, \mathrm{V}$

Preface $\ldots \ldots \ldots \ldots \ldots \ldots \ldots \ldots \ldots \ldots \ldots \ldots \ldots$ VII

1. Structure of Skin and Hair Follicles $\ldots \ldots \ldots \ldots \ldots \ldots \ldots \ldots \ldots \ldots$

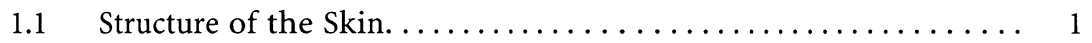

1.2 Structure of the Hair Follicle $\ldots \ldots \ldots \ldots \ldots \ldots \ldots \ldots \ldots \ldots \ldots \ldots$

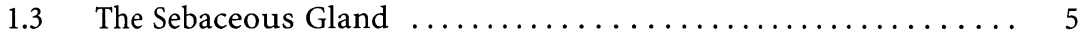

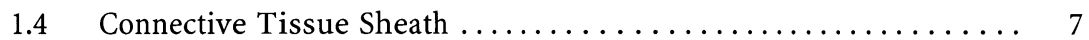

1.5 Dermal Papilla ............................ 8

1.6 Regional Anatomy of the Scalp.................. 13

2. Preparation of Thick Tissue Specimens . . . . . . . . . . . . . . 17

$2.1 \quad$ Development of Cellotape Method . . . . . . . . . . . . . . 17

2.2 Development of Cellophane Sheet Method .............. 18

2.3 Detailed Observations of Thick Tissue Specimens-Preparation of Microscopic and Electron Microscopic Specimens from Thick Tissue Specimen Using Cellophane Sheet ............. 20

3. Generation and Growth of Human Hair . . . . . . . . . . . . . . 23

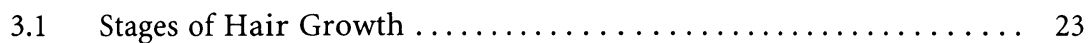

3.2 The Hair Canal ............................ 32

4. Pigmentation of Hair $\ldots \ldots \ldots \ldots \ldots \ldots \ldots \ldots \ldots \ldots \ldots \ldots \ldots \ldots \ldots \ldots \ldots$

$4.1 \quad$ Hair Color .................................... 33

4.2 Embryonic Development of Melanocytes in Human Hair . . . . . . . . 33

4.3 Generation of Melanin Pigmentation................ 34

4.4 Regeneration of Melanin Pigmentation ............... 35

4.5 New Growth of Melanin Pigmentation. . . . . . . . . . . 37

5. Blood Vessel System in Hair Follicles. . . . . . . . . . . . . . . . . . . . 39

5.1 Vascular System for the Pilosebaceous Unit .............. 39

$5.2 \quad$ Experimental Research........................ 40

5.3 Mechanism Underlying Formation of Vascular System in the Hair Follicle . . . . . . . . . . . . . . . . . . . . . 43

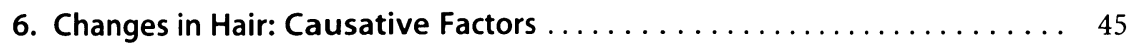

6.1 Hormonal Control of the Hair Follicle . . . . . . . . . . . . . . 45

6.2 Hormonal Control of the Sebaceous Gland ................ 45

6.3 Interaction Between the Sebaceous Gland and the Hair Follicle . . . . 46

6.4 Development of Axillary Hair.................. 47 


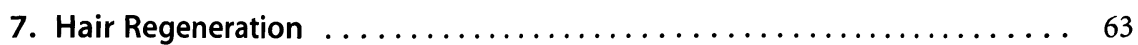

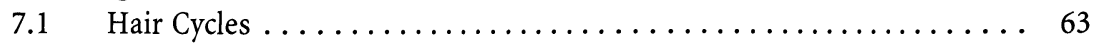

7.2 Mechanisms of Regulation of the Hair Cycle ............. 67

7.3 New Concept of Hair Generation and Regeneration .......... 67

8. Regeneration After Subcutaneous Tissue Shaving Procedure . . . . . . . 69

8.1 Common Hair Cycle Theory Reconsidered ................. 69

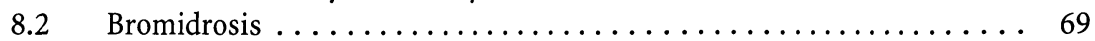

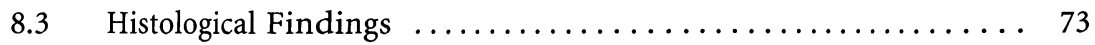

8.4 Relationship of Development Stage of New Hair Follicle and Size of Sebaceous Gland ....................... 81

8.5 Relationship Between New Hair Follicle and Time Elapsed

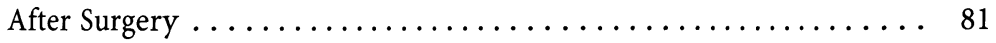

8.6 Evidence of New Hair Regeneration. . . . . . . . . . . . 81

9. Regeneration After Plucking $\ldots \ldots \ldots \ldots \ldots \ldots \ldots \ldots \ldots \ldots \ldots \ldots$

9.1 Process of Hair Regeneration After Plucking . . . . . . . . . . . 85

9.2 Summary of Our Findings................... 96

9.3 Process of Hair Follicle Formation After Subcutaneous Tissue

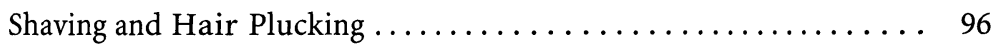

10. Regeneration After Electrical Epilation of the Hair . . . . . . . . . . . . 99

10.1 Basic Principles of Electrolysis and Electrocoagulation ......... . 99

10.2 Supportive Findings for Basic Principles of Epilation Treatment. . . 100

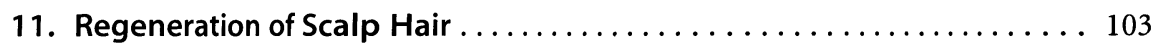

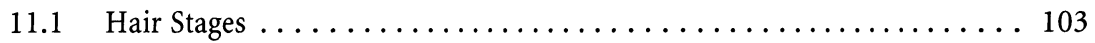

11.2 Supporting Findings........................ 107

12. Hair Regeneration in the Mouse After Application of Anti-Cancer

Agent, Particularly in Terms of Epithelial-Mesenchymal Interaction . . . 109

12.1 Anti-Cancer Agents and Hair Loss ... . . . . . . . . . . . . . . . . . . . 109

12.2 Regeneration of Hair Follicle After Application of Anti-Cancer

Agent ............................... 109

12.3 Regeneration from Remaining Bundle Telogen Hair Follicles. . . . . . 113

12.4 Regeneration from the Upper Isthmal Portion After the

Plucking of Telogen Hair . . . . . . . . . . . . . . 116

12.5 Regeneration After Destruction of the Sebaceous Gland ......... 116

12.6 Hair Regeneration in the Mouse................. 117

13. New Concept of the Hair Cycle (Sebaceous Gland Hypothesis) . . . . . . 119

13.1 Common Hair Cycle Theory Subject to Revision .............. 119

13.2 New Concept of the Hair Cycle in Humans ... . . . . . . . . . . . 119

13.3 Essential Hair Cycle Hypothesis and Supportive Findings . . . . . . 121

14. Other Important Findings in the Hair Follicle . . . . . . . . . . 123

14.1 Location of the Central Portion of Hair Regeneration ......... . 123

14.2 Mechanism of the Descending Hair Follicle . . . . . . . . . . 130

14.3 How Is the Hair Matrix Formed? ................. 133

14.4 Comparison of Epidermal and Follicular Cells ............ 135

14.5 Why the Growth (Anagen) Stage and Regressive (Catagen) Stage

Are Confused ............................ 137

15. Hormones Classified According to Secretory Organ of Origin ....... 143

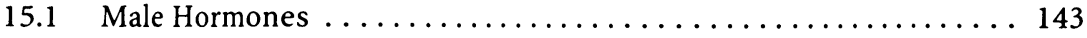

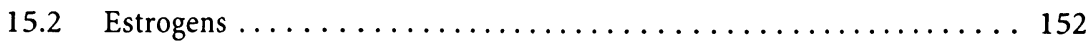

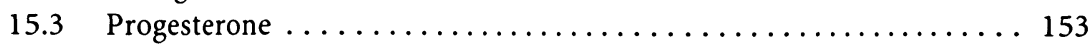

15.4 Distribution of Estrogen and Progesterone Receptors in Scalp Tissue 153

15.5 Adrenal Steroid Hormones ..................... 155

15.6 Thyroid Hormone. . . . . . . . . . . . . . . . . . . . 156

15.7 Anterior Pituitary Gonadotropins . . . . . . . . . . . 156 


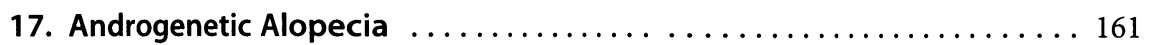

17.1 Definition of Androgenetic Alopecia . . . . . . . . . . . . . 161

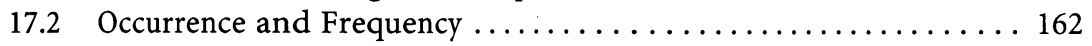

17.3 Incidence of Baldness in Japanese . . . . . . . . . . . . . . 163

17.4 Hair Density in the Scalp . . . . . . . . . . . . . . . . . 165

17.5 Factors Affecting the Hair Cycle . . . . . . . . . . . . . . . . . . 165

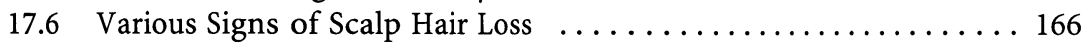

18. Etiologic Factors in Male Pattern Baldness . . . . . . . . . . . . . . . . 169

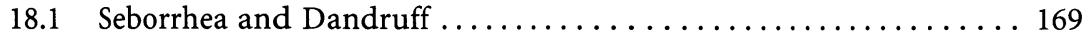

18.2 Dietary Factors . . . . . . . . . . . . . . . . . . . . . 169

18.3 Genetic Predisposition Theory .................. 170

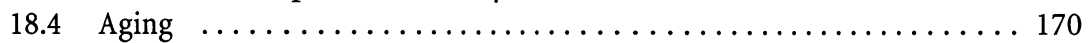

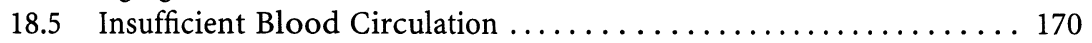

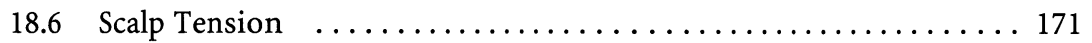

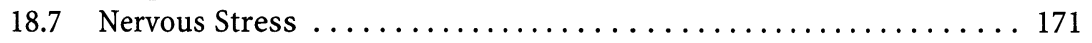

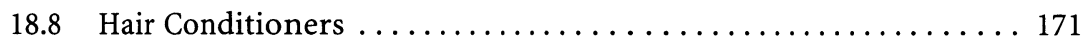

18.9 Hormonal Factors . . . . . . . . . . . . . . . . . . . . . . . 172

19. Sebaceous Gland Hypothesis of Androgenetic Alopecia (Inaba 1985; Inaba and Inaba 1992a) ............................ 175

19.1 Review of Hormonal Mechanisms in Hair Regeneration . . . . . . . . 175

19.2 Development of the Sebaceous Gland Hypothesis . . . . . . . . . . . 175

19.3 Mechanism of the Development of Androgenetic Alopecia . . . . . . 176

19.4 Hair Follicles and Sebaceous Glands Relative to 5 $\alpha$-DHT Production 176

19.5 Sebaceous Gland Hypothesis for Male Pattern Baldness;

Supportive Findings . . . . . . . . . . . . . . . . . . . 177

20. Histological Findings in Androgenetic Alopecia . . . . . . . . . . . . . 179

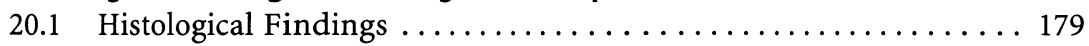

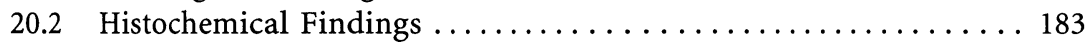

21. Causal Mechanism of Progressive Androgenetic Alopecia ......... 185

21.1 Fetal Period ................................... 185

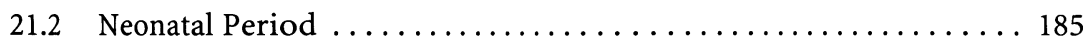

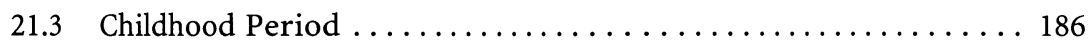

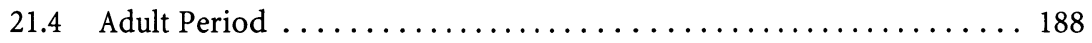

21.5 Balding Period (Solitary Coarse Hairs) $\ldots \ldots \ldots \ldots \ldots \ldots \ldots \ldots$

21.6 Complete Baldness .............................. 189

22. Differential Diagnosis (Types of Baldness Other than Male Pattern

Baldness) ................................... 191

22.1 Alopecia Areata . . . . . . . . . . . . . . . . . . . 191

22.2 Alopecia Cicatrisata . . . . . . . . . . . . . . . . . . . 195

22.3 Alopecia Gradus Induced by Hairstyling . . . . . . . . . . . . . . 195

22.4 Baldness Due to Syphilis ....................... 196

22.5 Other Contributory Diseases in Baldness . . . . . . . . . . 196

23. Relationship of Diet to Androgenetic Alopecia . . . . . . . . . . . 197

23.1 Malnutrition and Androgenetic Alopecia ................ . 197

23.2 Relationship of Animal Fat Intake to Androgenetic Alopecia . . . . 197

23.3 Japanese Diet . . . . . . . . . . . . . . . . . . . . 199

23.4 Relationship of Diet to Baldness and Hircismus (Offensive Body

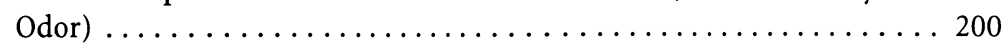

23.5 Diet Therapy for Androgenetic Alopecia . . . . . . . . . . . . 201

24. General Measures for Prevention of Baldness . . . . . . . . . . . . . 205

24.1 Prevention of Baldness by Measures That Promote Healthy Scalp

Hygiene . . . . . . . . . . . . . . . . . . 205 
25. Therapy for Androgenetic Alopecia . . . . . . . . . . . . . . . . . . . 209

25.1 Increased Massage and Circulation $\ldots \ldots \ldots \ldots . \ldots . \ldots . \ldots 209$

25.2 Diet Therapy ............................ 210

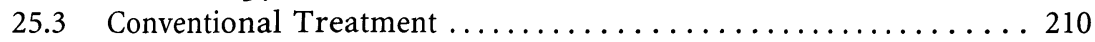

26. Agents Used for the Treatment of Androgenetic Alopecia . . . . . . . 211

26.1 Background and Historical Aspects of Agents Used for the

Treatment of Androgenetic Alopecia . . . . . . . . . . 211

26.2 Treatment of Androgenetic Alopecia with Steroidal

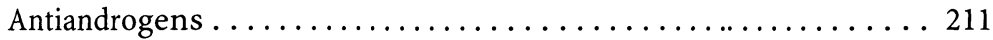

27. Other Products Being Developed by Pharmaceutical and Cosmetic

Companies ............................... 215

27.1 Treatment of Androgenetic Alopecia with Chemical Products . . . . 215

27.2 Chemical Structure of These Products . . . . . . . . . . . 216

27.3 Mechanism of Hair Growth in Relation to New Drugs .... . . . . 216

27.4 Mechanism of Action of New Drugs on Target Tissue . . . . . . . 217

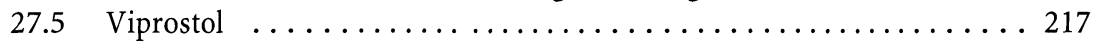

27.6 Other Products in Development .................. 217

28. Trial Treatment of Androgenetic Alopecia with Oxidizing Agents (Inaba

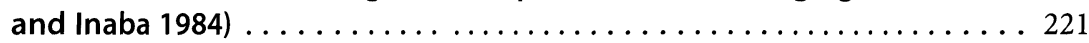

28.1 The Hair Growth-Stimulating (Trichogenous) Effect of Cold Wave

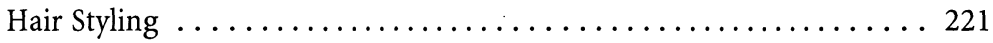

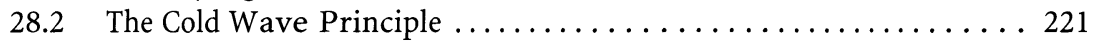

28.3 Basis of Hair Regeneration ...................... 221

28.4 Discovery of an Effective Trichogen .................. 222

28.5 Development of New Tonic Formulation . . . . . . . . . . . 223

28.6 Treatment of Androgenetic Alopecia with Stabilized Chlorine

Dioxide (Inaba 1985) ....................... 224

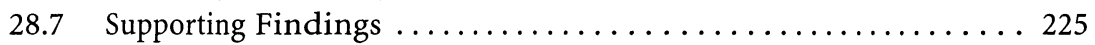

28.8 Individual Case Reports of Patients with Androgenetic Alopecia

Treated with the Inaba Reagent $\ldots \ldots \ldots \ldots \ldots \ldots \ldots \ldots \ldots \ldots \ldots \ldots \ldots \ldots$

28.9 Classification of Active Principles of Hair Tonics by Mechanism of

Action (Reviewed by Imada 1983) . . . . . . . . . . . . . . . . . 229

28.10 Future Aspects of Hair Care Products . . . . . . . . . . . . 232

28.11 Trichogenous Agents in the United States . . . . . . . . . . 232

29. Operative Treatment for Androgenetic Alopecia ............. 233

29.1 Punch Graft Method ........................ 233

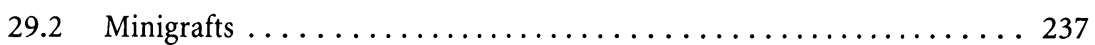

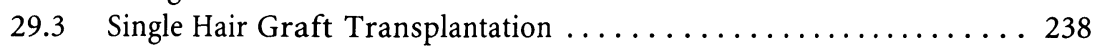

29.4 Multiple Strip Composite Graft ................... 244

29.5 Histological Findings After Transplantation of Single Hair-Bearing

Graft or Micrograft ....................... 244

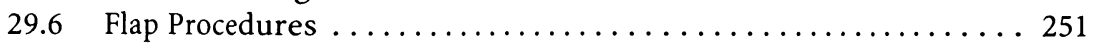

29.7 Scalp Reduction Procedures ....................... 262

29.8 Scalp Skin Elongation Method ..................... 264

29.9 Signs of Disturbed Circulation with Juri Flap Methods . . . . . . . . 267

29.10 Advantage and Disadvantages of Juri and Partial Flaps . . . . . . 267

29.11 Free Flaps . . . . . . . . . . . . . . . . . . . . . 267

29.12 Histological Study of Free Coarse Hair-Bearing Graft

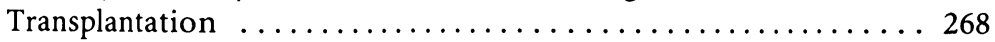

29.13 Marechal Operation (Bilateral Ligation Method) ............ 275

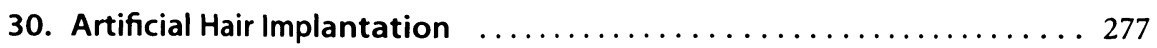

30.1 Artificial Hair Implantation . . . . . . . . . . . . . . . . . . 277

30.2 Our Experience of Artificial Hair Implantation . . . . . . . . . . 278

30.3 Artificial Hair Newly Developed by Nido Ltd. . . . . . . . . . . . 280

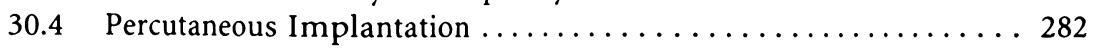

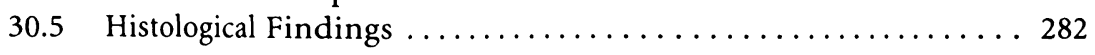

XII Contents 
30.6 Case Reports . . . . . . . . . . . . . . . . . . . . . . . . 283

30.7 Mechanism of Epilation After Implantation of Artificial Hair . . . . . 284

30.8 Advantages of Artificial Hair Implantation . . . . . . . . . . . . . . 285

30.9 Drawbacks of Artificial Hair . . . . . . . . . . . . . . 286

30.10 Other Problems Related to Artificial Hair ............... 286

31. Another Solution: Hairpieces (Wigs) $\ldots \ldots \ldots \ldots \ldots \ldots \ldots \ldots \ldots . \ldots \ldots$

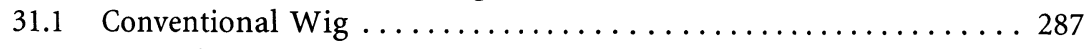

31.2 New Wigs . . . . . . . . . . . . . . . . . . . . . . 289

32. Mechanism of Linear Scar Formation After Scalp Surgery: Preventive

Measures ................................ 291

32.1 Case Studies . . . . . . . . . . . . . . . . . . . . . 291

Afterword ................................... 299

Acknowledgments ........................... 300

References ............................. 301

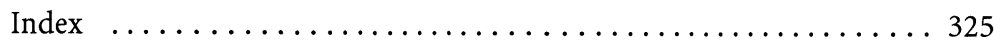

